\title{
Animation for Validation of Business System Specifications
}

\author{
V. Lalioti \\ GMD- German National Research Center for Information Technology, \\ Schloss Birlinghoven, \\ 53754 Sankt Augustin, \\ Germany \\ e-mail :vali.lalioti@gmd.de
}

\begin{abstract}
Business System Engineers, responding to changes in the market place, are faced with the challenge of building increasingly complex and varied systems. Formal approaches and modeling tools, incorporated in the CASE technology, are used to aid the Requirements Engineering (RE) activity, which leads to a high level specification of Business Systems. The Validation of these specifications is a very delicate activity since it requires heavy stakeholder involvement and a consensus between stakeholders and analysts, who have quite different backgrounds and concerns. The Validation approach that is put forward in this paper, uses a set of conceptual modeling formalism and a set of formal scenarios, together with a mechanism to automatically generate them. In addition, the approach makes use of Animation techniques in order to visualize the scenarios. A system that implements the approach is also described in this paper.
\end{abstract}

\section{Introduction}

Business System Engineers, responding to changes in the market place, are faced with the challenge of building increasingly complex and varied Business systems. Formal approaches and modeling tools, incorporated in the CASE technology, are used to aid the Requirements Engineering (RE) activity, which leads to a high level specification of Business Systems
[1]. These specifications will be then used to formally specify the Information System, which could support part or the whole of the Business processes. It is widely accepted that the Validation of these specifications early in the Business Systems life cycle will save customer organizations both time and money [2].

The Validation of requirements specification is a very delicate process, since it requires interaction, communication and a consensus between stakeholders, as well as between stakeholders and analysts, who have quite different backgrounds and concerns. The approach that is put forward in this paper, uses a set of conceptual modeling formalisms for business system specification and a set of formal scenarios which are closely linked with the specifications. The Business system Specifications are probably going to change many times during the RE process and this approach provides the means of validating and evaluating the effect of the change without any coding-effort or extensive prototyping.

The way the validation scenarios are communicated to the stakeholder is very crucial. The Validation approach that is put forward in this paper, makes use of Animation techniques in order to visualize the set of formal scenarios. In this context, the paper presents the validation approach and a tool for supporting the approach. The requirements and an overview of the proposed approach are given in section 2 . Then the validation scenarios and the way there are visualized are described, in sections 3, 4 
respectively. A system for supporting the approach was implemented and its functionality is demonstrated in section 5. The paper concludes, by summarizing the basic characteristics of the approach and the possible future enhancements.

\section{Animation for Validation - Requirements and Overview}

Business Systems Engineering is the activity that transforms the needs and wishes of the stakeholders, which are usually incomplete and informal, into complete and consistent business system specifications, preferably written in a formal notation.

Current CASE tools and existing methodologies use conceptual modeling formalisms and visual representations of them to improve the process of verifying the well-formness and correctness of the specifications. However, there is no possible proof that the verified specifications address the elusive user's needs. Additional support is needed for the validation phase of the RE process. But it is not easy to make specific recommendations about how to improve validation practices, because there is little hard evidence about exactly what analysts, developers and marketing people do. Computer-supported or automated techniques used for validation include executable specifications [3], rapid prototyping [4] and animation [5]. Such techniques are able to provide an indication of the dynamic behavior of the system under development, but involve extensive coding effort or force the Business System Engineers to make some design decisions prematurely.

In the Lubars review of the state of practice in requirements modeling [6] was found that, although requirements validation is both a technical and organizational problem, the only technique in universal use is one organizational in nature: the review-meeting. During reviews, stakeholders appear to be most interested in threads or scenarios. It was also observed that models of system behavior based on stimulus-response couplings seem to have a significant advantage over other representations: they engage the stakeholder 's interest and relate the system description to the stakeholder 's knowledge of the Business domain.

A variety of systems and approaches follow the above observations and use scenarios in various phases of the Requirements Engineering process. For example, in requirements elicitation scenarios are a behavioral specification, while in scenario analysis, scenarios are possible ways to use the system to accomplish some function the user desires. In validation, scenarios aim at demonstrating how the system will work once it is in operation. The results drawn from the Lubars study as well as the study of existing systems that use scenarios, were particularly promising.

Recently, visualization techniques, which have been successfully used in programming and data visualization [7] [8] [9], have been also employed in various phases of the RE process [5] [10]. For example, Task/Actor simulation has been combined with animation for the dynamic modeling of organizations in [11].

This paper advocates that many benefits can be accrued by the application of animation techniques in the validation process; thus integrating the formality of conceptual models and scenarios with the interactive and user-friendly technology of animation. In addition, changes in the Specifications can be easily validated and their impact on the overall Business System could be evaluated by automatically generate and re-execute the same scenario, after the changes have been made on the Business System Schemata.

A diagrammatic overview of the approach is given in figure 1. The conceptual models and metamodels are stored in a common Repository. Three models, developed as part of the ORES $^{1}$ ESPRIT project [12], are used to capture the static and dynamic aspects of the Business System. The Scenarios Engine is the basis of the approach and is responsible for gathering and structuring information from the Repository in order to form the Validation Scenarios.

The Scenarios Engine is also responsible for executing the scenarios and reporting all the interesting events and information to the Animation Engine to be visualized.

The Animation Engine is the component responsible for animating the execution of a scenario.

1 The ORES project is a collaborative project between: 01 Pliroforiki, Greece; Clinica Puerta de Hierro, Spain; Information Dynamics, Greece; Royal Institute of Technology, Sweden; UMIST, U.K. The project is partly funded by the CEC under the ESPRIT III programme. 
It is also responsible for transferring any user requests to the Scenarios Engine. The Animation Engine generates and updates simultaneously the Visual Views according to the events reported by the Scenarios Engine. A Visual View consists of a physical window and a set of graphical objects which are displayed within the window. Each View is sensitive to a set of graphical events, such as resizing and redrawing parts of the View's window whenever this is necessary. Three Visual Views are generated by the Animation Engine, one for each conceptual model. be validated by selecting a particular scenario to execute. Furthermore, if a change in the Business requires changes in the system, then only the Business System Schema has to be changed. Then by observing the execution of the same scenario, an indication of the impact of the change to other parts of the Business System can be evaluated and validated.

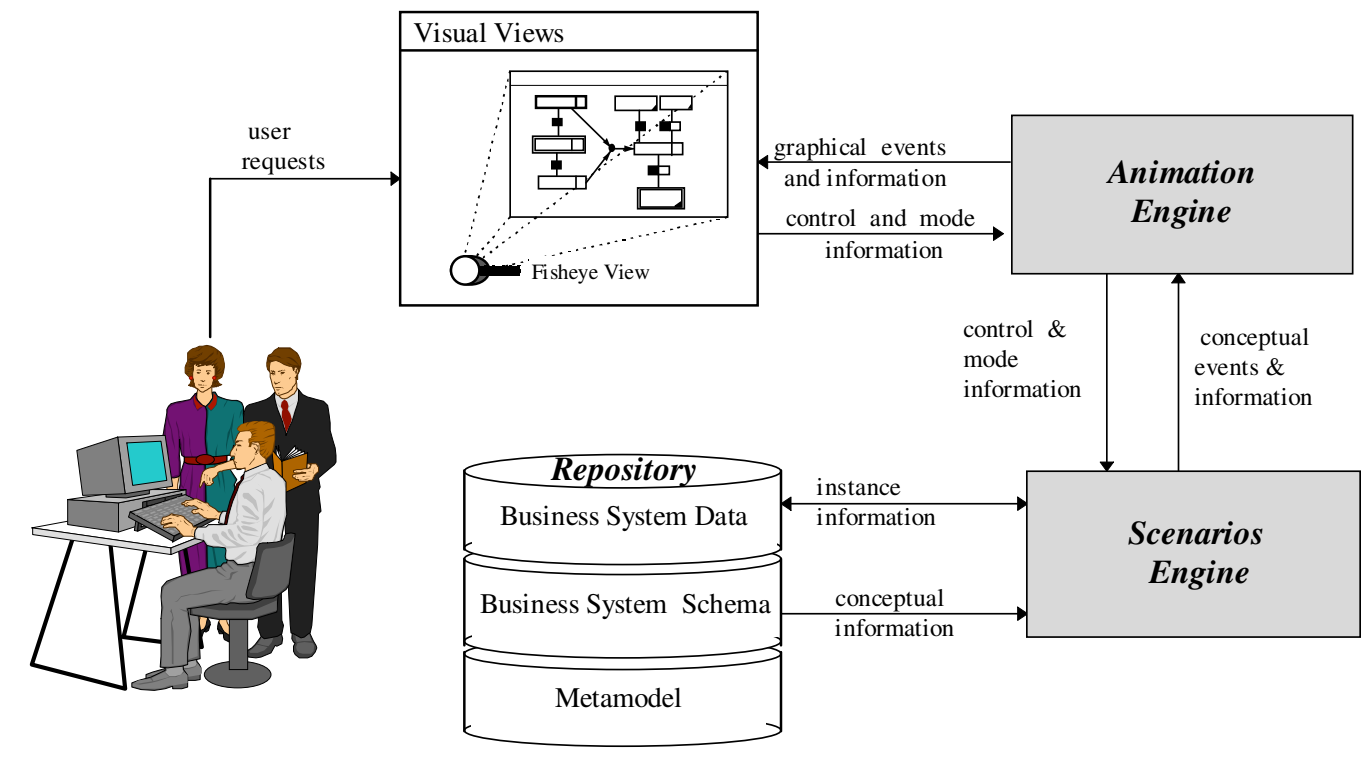

Figure 1. An Architecture for Animation for Validation

The stakeholder assists the Business System engineer in choosing a particular scenario and finding errors and omissions by observing and controlling the execution of the selected scenario. However, the user directly interacting with the tool is expected to be the Business System engineer. In case the stakeholder wishes to interact with the system can be helped by the Business System engineer in doing so. Therefore, the term user is used in this paper to denote both of them.

Business System specifications are likely to change a lot during the RE process. In our approach once a Business System Schema has been developed it can

\section{The Validation Scenarios}

For the task of enterprise modeling it is important to capture both the structural as well as the behavioral aspects of the domain.

Therefore, the conceptual modeling language which is used provides mechanisms for three conceptual views namely a structural view, a behavioral view and an active view, as shown in Figure 2. These three views are represented by the ERT, PID and CRL models respectively. Apart from these models, a query language which operates on ERT objects has been defined. The ERT-SQL 


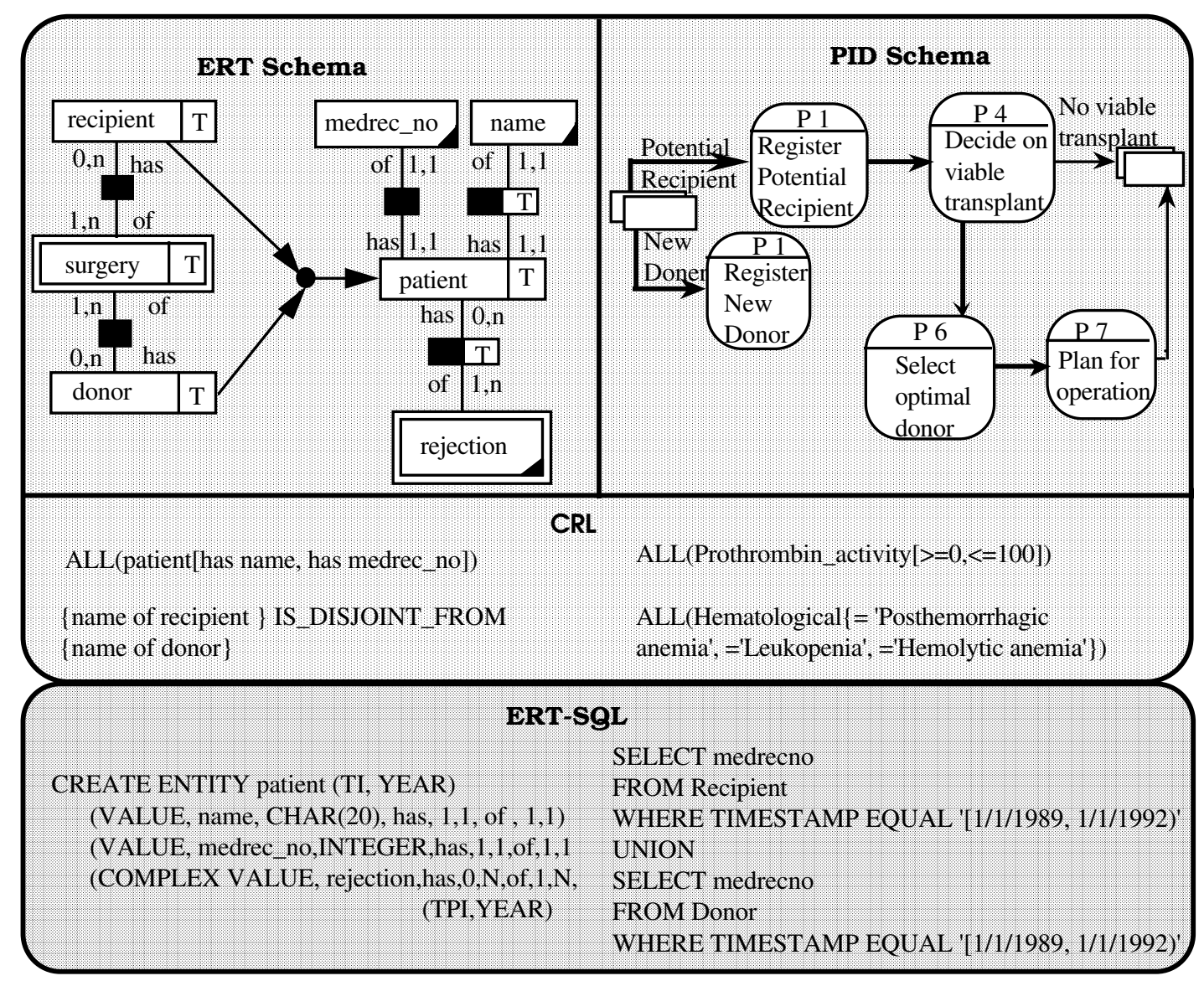

Figure 2. Example of a Structural, Behavioral and Active view of a BS Specification

language provides the means of manipulating ERT data, i.e. queering and retrieving data. The language is based on the ERT Algebra [13].

During Validation is important to be able not only to view the structural and behavioral aspects of the Business domain separately, but also observe the relationship between the two.

Therefore, a major reason for using the particular modeling formalisms is their close interrelationship, which is explicitly recognized and represented according to the metamodels of these formalisms. A Validation Scenario follows these interrelationships in order to walk through the Business System schemata in a well structured way. As illustrated in Figure 3 for example, a process could refer to or affect an ERT object and can be initiated either by another process, or an external agent.

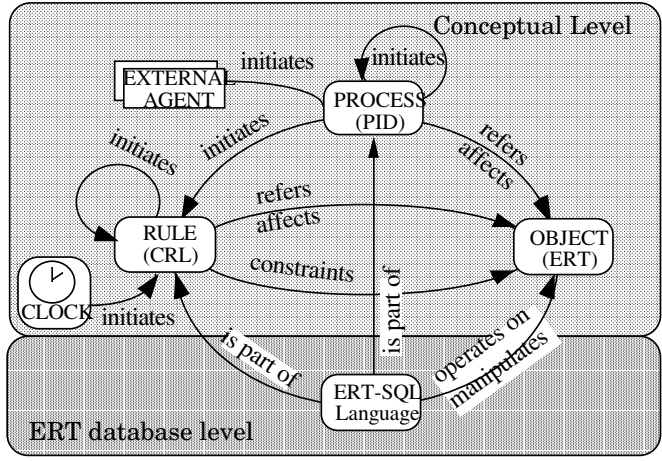

Fig. 3. Interrelationships of the Conceptual Models

The main innovation of the approach, that is put forward in this paper, is that the scenarios are formal and automatically generated. Scenarios are generated by combining together a set of formal questions in a 
number of meaningful ways. In particular, a set of questions could be asked, which all have one or more of the conceptual models as their starting point. Some examples of questions follow:

(1) What if event $X$ arrives at the system, which shows which processes are initiated by event $\mathrm{X}$. This is the main means of demonstrating system's behavior.

(2) What are the operations performed by external agent $\mathrm{A}$, which shows what are the tasks of an external agent. This could clarify the operations performed by different groups of users.

(3) Which processes or rules are initiated by process $\mathrm{P}$, which gives all the processes or rules that could be triggered by the execution of process $P$. This could provide information on the general rules which apply to the Business and their relationship to specific processes.

(4) Which objects are directly linked to object E by a relationship or an ISA relationship, which gives a subpart of the ERT schema with object E in focus. This could provide an insight to the structural part of the business system.

(5) Which object instances satisfy the preconditions of process $\mathrm{P}$, which provides instances that satisfy the precondition of a process. This could be used to either automatically execute a process or to provide fragmentary explanatory information about a process.

(6) Which processes or rules affect a particular object, which determines the effect rules and processes have on an object. This is particular useful when checking the way objects are modified and changed by the system.

Most of the questions could be directly used to generate explanatory scenarios, while others could be combined together to form process scenarios. Examples of validation scenarios are given below:

\section{Scenario 1}

This scenario has the PID model as the starting point and the first question asked is: what if event $\mathrm{X}$ arrives at the system (question 1). Then the processes that would be initiated by that event are found and the user could choose one to execute. The process will be executed and the ERT objects affected by it will be presented (question 6). Also the rules or processes which are initiated by the output events of the executed process are given to the user (question 3 ), who could either terminate the scenario or continue by selecting a process or a rule from the new set to follow.

\section{Scenario 2}

This scenario is concerned with the user groups performing the tasks, and checks whether tasks are assigned to external agents properly. The scenario starts with the question: what processes are performed by external agent $\mathrm{A}$ (question 2). This question will result a set of processes that could be initiated by a particular external agent. Then the user can either choose one process and then use scenario 1 , or terminate the scenario.

\section{Scenario 3}

This scenario is concerned with the ERT model only and provides a better understanding of how entities are related to each other. The Scenario starts with the question which ERT objects are linked to ERT object E, by an ISA relationship or by any direct relationship (question 4). After the first level of relevance is examined the user could continue by asking which entities are linked with an indirect relationship with object $\mathrm{E}$ or choose to terminate the scenario.

Most of the automated techniques that use scenarios, provide mechanisms for interacting and controlling the execution of a scenario. However, this interaction is mostly concerned with choosing between different execution paths and providing any additional information needed for the execution. This paper argues that providing different ways of viewing and controlling the execution of the scenarios, both in terms of what and in terms of how information is presented, would greatly improve the understanding of the specified Business System requirements, thus facilitating the validation process. This is achieved by providing mechanisms for abstracting and zooming on the Business System schemata, during the execution of a scenario.

Three different abstraction levels have been chosen, namely the conceptual level/ERT instance level, the single model/intermodel and the explanatory/process. The first pair of abstraction levels allows to observe the execution of a scenario either at the high level of the conceptual models or at the very detailed one which uses specific instances of Entities. There are also occasions in which it is better 
to observe only one model during the execution of a scenario, or involve more than one; thus the second pair of abstraction levels. Finally, the possibility of executing only one step of a scenario is provided by the last abstraction level.

In addition, three "zoom" mechanisms are defined for concentrating only on some part of a Business System schema, namely the hierarchical zoom, selective zoom and dialog-based zoom. In hierarchical-zoom, the various concepts can be examined at different levels of detail, while in selective-zoom, concepts are layered in terms of levels of importance. In dialog-based zoom the use of dialogs allows the user to specify the concepts that are of interest. These mechanisms can be used for all the conceptual models. However, in our approach they are defined only for the ERT model, which is the most complex one and it was found necessary for additional abstraction mechanisms to be introduced.

\section{The Visualisation of Scenarios}

The scenarios are used to structure the information that is presented to the user and the abstraction/zoom mechanisms provide the necessary control mechanism for executing a scenario at different levels of detail. In terms of presenting the execution of the scenarios to the user, two are the basic requirements: the representation should facilitate the communication of the structured information between the actors of the RE process and it should reflect the dynamic aspects of the specified system.

According to the above requirements the approach uses graphics, color and movement to animate the execution of the scenario at real time. The component responsible for the visualization is the Animation Engine. It receives conceptual events and information and translates them into graphical ones. Three Visual Views, one for each conceptual model, are generated by the Animation Engine, to visualize different aspects of the validation scenarios. The Animation Engine is responsible for controlling the Views and displaying the appropriate graphical objects on each View. Each View is updated simultaneously during the execution of a scenario.

The use of parallel animated views to visualize the execution of a scenario provides a more accurate and uniform view of the specifications as a whole. However, in case of very large or complex Business
System schemata (e.g. large ERT schemata), it would be desirable to further enhance the visualization approach by providing different visualization mechanisms within a Visual View.

Three visualization mechanisms have also been defined, namely the Iconic, Diagrammatic and the Fisheye. In the Iconic visualization mode, the ERT View for example, displays only the top level of the ERT model, using an icon for each entity or value class. Figure 4, gives an example of an iconic view of the ERT subschema concerning the Patient Entity. This iconic view could also be used together with the fisheye mechanism. The Diagrammatic visualization mode for the ERT View is the most detailed one. A fragment of an ERT schema is displayed, with the diagrammatic format used in ERT diagrams.

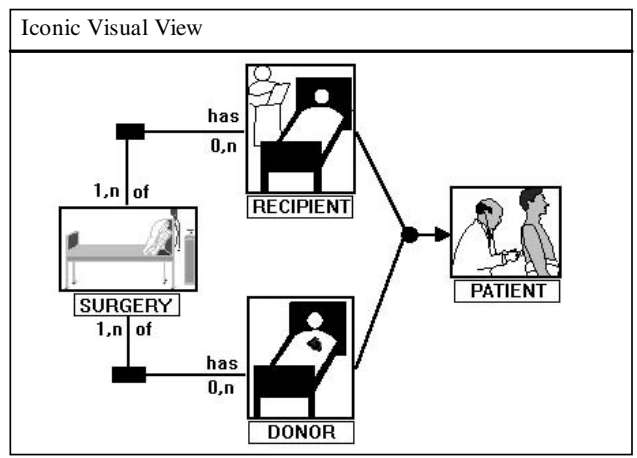

Figure 4. An Iconic Visual ERT View

The Fisheye visualization mode provides a more comprehensive view of a fragment of a Business System schema. Fisheye Views use strategies to display details near a focal point and only more important landmarks further away [14]. Conceptually, these views are implemented by computing a degree-of-interest value for every object, then displaying only those objects that have a degreeof-interest value greater than a criterion [15]. The approach uses similar techniques to provide a view of a fragment of the ERT Business System schema, where the entity of interest is the focal point, and the rest of the ERT objects are displayed further away depending of their degree of "relevance" with the selected entity. 


\section{Example}

A system that implements the approach was developed using the $\mathrm{C}$ programming language and the PHIGS 3-D graphics tool. The repository which holds the metamodel and the Business System schemata and data was implemented by the ORES consortium using the INGRES Data Base Management System.

The case study used in this paper is a small subset of the ORES Case Study. This subset cannot serve as a complete business case. However, the purpose of its use in this paper, is to demonstrate the way different scenarios and animation mechanisms facilitate the validation process, by exploring specific aspects of the business schemata.

It is important to note that the system uses a three dimensional space and 3-D graphical objects to represent the Conceptual Models. For example, in the PID View, processes are visualized by a 3-D box, external agents by a sphere and flows by a line stating from the center of one 3-D object to the center of another. The textual graphical objects are also placed in a 3-D space. In addition the positioning of the graphical objects during the execution of a scenario in the Views is automatically managed by the Animation Engine.

The user of the system can select the scenario to execute and the mode of execution via a control panel. A typical session with the system is composed of selecting the scenario, setting the modes of execution, choosing the conceptual object with which the scenario will start the execution, observing the visualization and animation of the scenario while controlling the speed of the execution. If it is found necessary to change parts of the Business System Schemata, then these are firstly made on the specifications and then the same validation scenarios could be animated in order to observe their effect.

\subsection{Scenario 2 - Process Scenario}

To demonstrate the way the approach is used to validate the tasks assigned to a particular external agent, we assume that the user selected scenario 2, using the PID and ERT Views and selected an External Agent as the starting point of the scenario.
The PID View reacts by displaying the selected agent. The next step is to find what triggers are generated by this external agent and then which processes are triggered by those triggers. The next step is to execute the processes and find the ERT objects that are affected. The system then displays these objects according to the zoom and visualization modes selected, in the ERT View. The scenario proceeds by finding the output events generated by the executed process and the processes triggered by those events. In figures 5 and 6, snapshots of the PID and ERT Views during the execution of validation scenario are given.

As shown in figure 6, every ERT object affected by a process is displayed in the ERT View with the same color that the corresponding process has in the PID View of Figure 4. In addition, when a new ERT object is displayed in the ERT View the relationships of this object with any other already displayed, are drawn with the same color of the object. By default only the immediate relationships are displayed.

\subsection{Scenario 3 - Explanatory Scenario}

This is an explanatory scenario that focuses on the ERT Model. The scenario starts with an ERT object that the user is interested in and displays any other ERT object related to it either by a relationship or an ISA relationship with level of relevance defined by the selective zoom mode setting. For this example, we assume that the user chose the entity PATIENT.

Then the ERT View displays all entities and value classes which are related to the selected entity via a direct relationship. If the selective zoom mode is set to be two, the ERT View then displays any entities or value classes related to the selected entity via a binary relationship with another entity/value class which is directly related to the chosen entity. The final result is shown in figure 7.

The system has the option of producing a Fisheye View of the part of the ERT Model which is of interest. The Fisheye View of this example is given in figure 8 . The view of Figure 8 was generated by a three step process. Firstly, the more important objects were positioned closer to the viewing point, while the ones with a first level of relevance are slightly further away, the ones with a second level of relevance even further back and so on. Secondly, a perspective projection was used to distort the objects so as the 
closer ones appear bigger while the ones further back smaller. Thirdly, depth queuing has been used to make the color of the objects fading depending on their position on the z-axis.

\section{Conclusions and Future Enhancements}

The approach presented in this paper, combines the use of formal scenarios with visualization and animation techniques, for the purpose of ensuring that the Business System specifications, developed during the requirements elicitation phase, really reflect the stakeholder 's needs and statements about the Business.

The main innovation of this approach is that it can be customized, both in terms of the scenario and in terms of the way it is presented to the user. In particular, scenarios can be automatically generated according to user preferences and different representation mechanisms could be combined together to visually present the execution of the scenario, in the most suitable way. In addition, changes in the organizational structures or processes, could be evaluated and validated by executing a particular scenario again, after changing the Business System Schemata to reflect the changes in the Business. During the re-execution of the scenario the impact of the changes to the other parts of the Business System could be observed in the animated Views.

A system that supports the approach has been used for validating a subset of the ORES Case Study, giving promising results. However, additional case studies should be used in order to assess the use of different visualization modes and to define the guidelines for managing the approach in terms of time and resources. Therefore, future research will concentrate on three main directions. Firstly, the use of visual objects for representing business processes and business data in a way that resembles the real life will be further explored. Secondly, a set of guidelines for the way the system should be used by the stakeholders and Business System Engineers would be defined and evaluated by applying it in different project and organizations. Finally, further research towards a definition of a complete set of questions for forming validation scenarios, would add to the flexibility of the system.

\section{References}

[1] Bubenko J., Rolland C., Loucopoulos P., DeAntonellis V., Facilitating "Fuzzy to Formal" Requirements Modelling, IEEE International Conference on Requirements Engineering, Colorado, pp. 18-22, April, 1994

[2] Boehm B.W., Verifying and Validating Software Requirements and Design Specifications, IEEE Software, Vol. 1, No. 1, January 1984.

[3] Henderson P., Functional Programming, Formal Specification and Rapid Prototyping, IEEE Transactions on Software Engineering, Vol. SE-12, No. 2, February 1986, pp. 241-249

[4] Johnson J.R., Prototyping, The Software Factory: Managing Software Development and Maintenance, 2nd ed., pp. 195-204, Computer Weekly, 1991.

[5] Tsalgatidou A. and Loucopoulos P., Rule-based behaviour modelling: specification and validation of information systems dynamics, Information and Software Technology, Vol. 33, No. 6, July/August 1991, pp. 425-432 [6] Lubars M, Potts C. and Richter C., A Review of the State of the Practice in requirements Modelling, Proc. Int. Symposium on requirements Engineering, 1993

[7] Bernstein D., Bolmaricich A. and So K., Performance Visualisation of Parallel Programs on a Shared Memory Multiprocessor System, Research Report, RC 14635 (\#64061) Computer Science, 1 September 1989.

[8] Baecker R.M., An Application Overview of Program Visualisation, Computer Graphics, 20, 4: 325, 1986.

[9] Brown M.H., Perspectives on Algorithm Animation, CHI'88: Human Factors in Computing Systems Washington, D.C., 33-38, 1988

[10] Lalioti V. and Loucopoulos P., Visualisation of Conceptual Specifications, Information Systems Journal, Vol. 19, April 3, 1994

[11] R.C.J. Dur and P.W.G. Bots, Dynamic Modelling of Organisations using Task/Actor simulation, In Dynamic Modelling of Information Systems II, H.G.Sol and R.L. Crosslin (Ed.), Elsevier Science Publishers, pp. 49-74, 1992.

[12] Theodoulidis, C., Loucopoulos, P.,Wangler, B., "A Conceptual Modelling Formalism for Temporal Database Applications", Information Systems, Vol. 16, No. 4, pp. 401-416, 1991.

[13] Karvelis G and Theodoulidis B., An SQL-like Language for the ERT Model, ORES project, Technical Report P7224 ORES/UMIST/WPE/2/v5, May 1994

[14] Mendelzon A.O., Declarative Database Visualisation: Recent Papers from the Hy+/GraphLog Project, Technical Report CSRI-285, University of Toronto, June 1993

[15] Sarkar M. And Brown M.H., Graphical Fisheye Views, Communications of the ACM, December 1994, Vol 37, No. 12. 


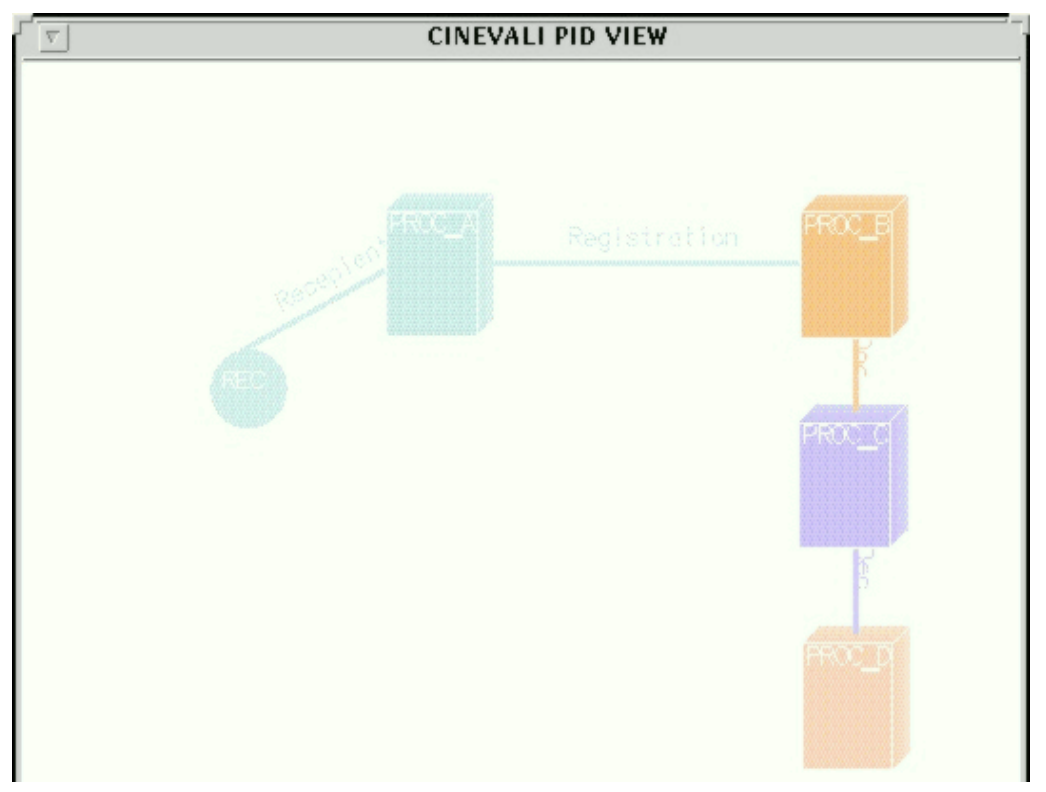

Figure 5. Snapshot of PID View during execution of scenario 2

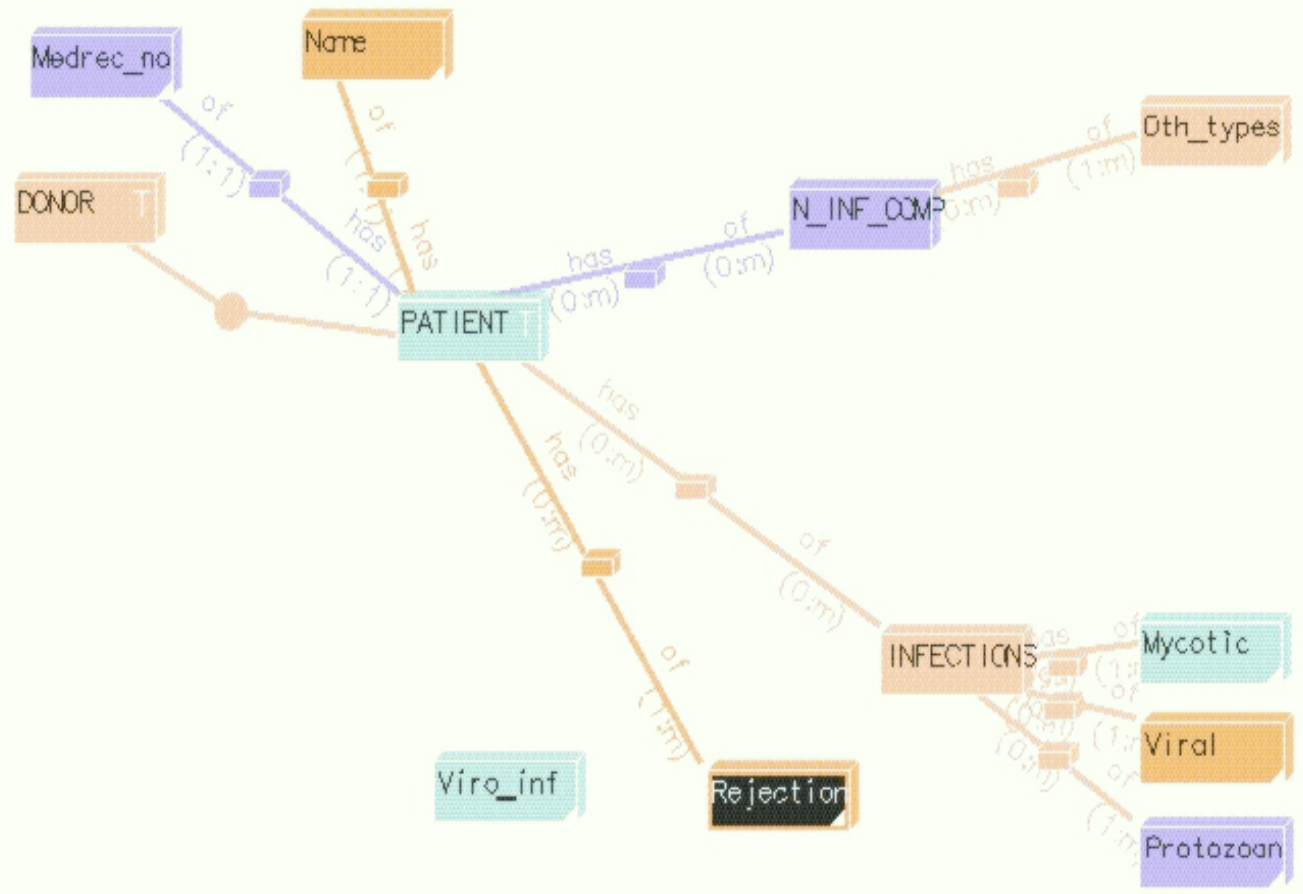

Figure 6. Snapshot of ERT View during execution of scenario 2 


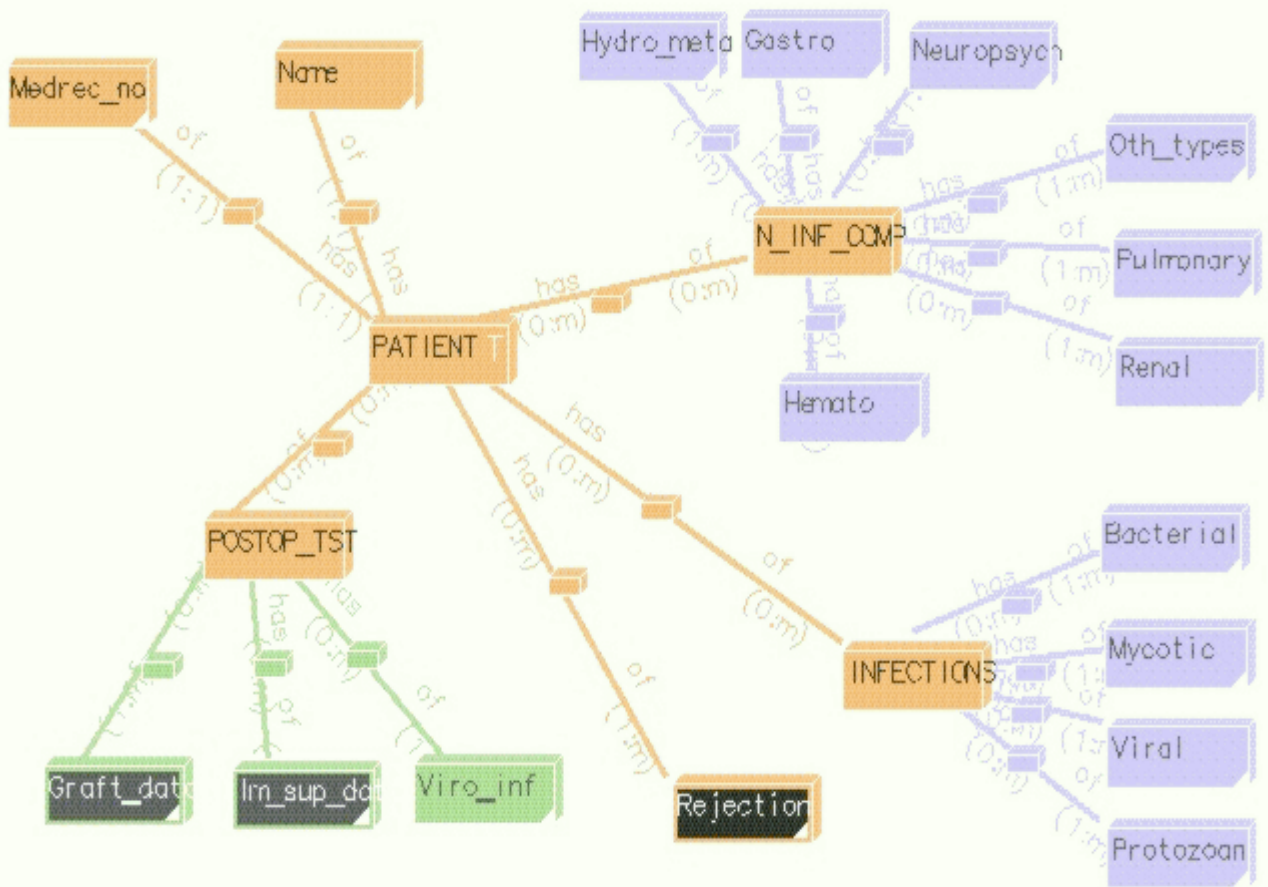

Figure 7. A Diagrammatic Visual ERT View

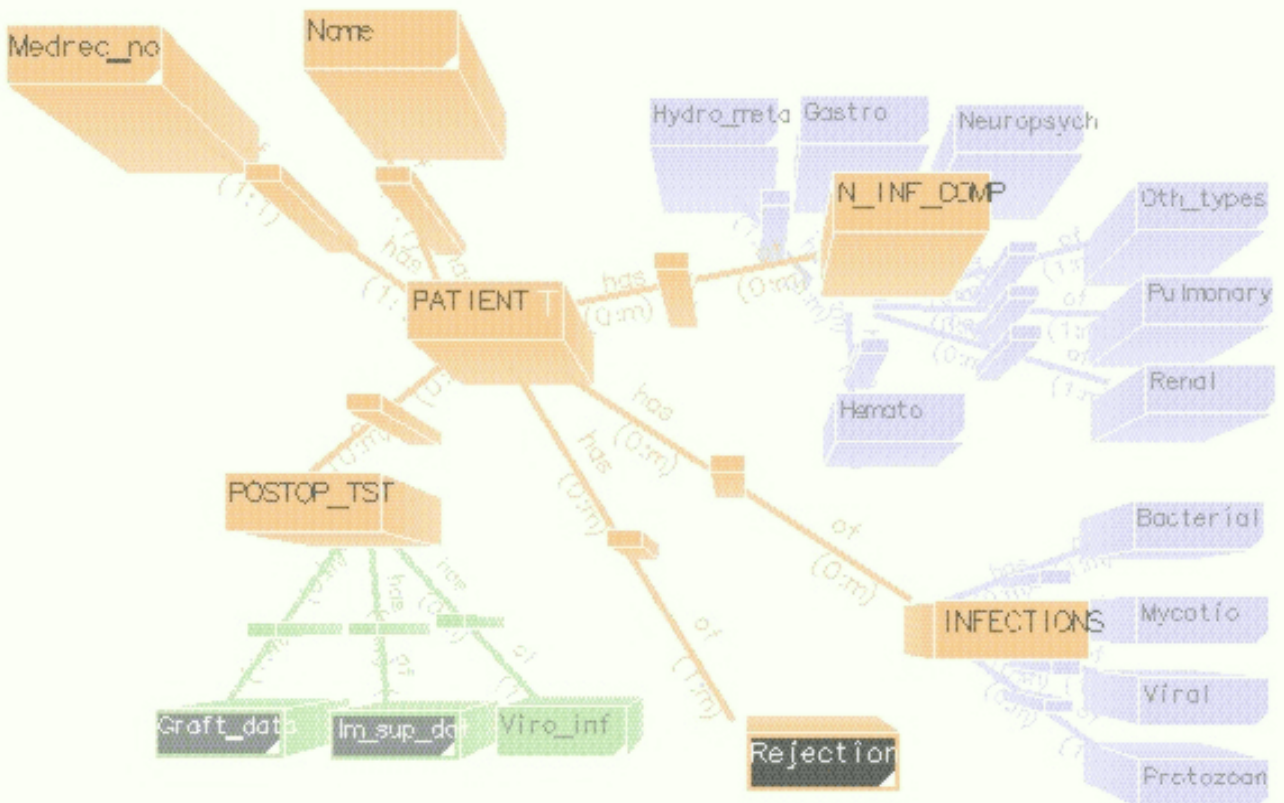

Figure 8. A Fisheye Visual ERT View 